\title{
Combinatorial Synthesis and Ionic Conductivity of Amorphous Oxynitrides in a Pseudo-ternary $\mathrm{Li}_{3} \mathrm{PO}_{4}-\mathrm{Li}_{4} \mathrm{SiO}_{4}-\mathrm{LiAlO}_{2}$ System
}

\section{Shunsuke SASAKI, ${ }^{\mathrm{a}, \mathrm{b}}$ (1) Atsuo ONO, ${ }^{\mathrm{a}}$ Akiyoshi SUZUKI, ${ }^{\mathrm{a}, \mathrm{s}}$ Masaki TAKEI, ${ }^{\mathrm{a}}$ Kota SUZUKI, ${ }^{c, \xi}$ (1) Masaaki HIRAYAMA, ${ }^{b, c, \S}$ (1) and Ryoji KANNO ${ }^{c, *, \S \S}$ (1)}

\author{
a ULVAC Inc., 2500 Hagisono, Chigasaki, Kanagawa 253-8543, Japan \\ b Department of Chemical Science and Engineering, School of Materials and Chemical Technology, Tokyo Institute of Technology, \\ 4259 Nagatsuta, Midori-ku, Yokohama 226-8502, Japan \\ ' Research Center for All-Solid-State Battery, Institute of Innovative Research, Tokyo Institute of Technology, \\ 4259 Nagatsuta, Midori-ku, Yokohama 226-8503, Japan \\ *Corresponding author: kanno@echem.titech.ac.jp
}

\section{ABSTRACT}

A combinatorial synthesis system consisting of co-sputtering multiple radio frequency (RF) cathodes was developed to investigate the presence of new amorphous solid electrolytes in a pseudo-ternary $\mathrm{Li}_{3} \mathrm{PO}_{4}-\mathrm{Li}_{4} \mathrm{SiO}_{4}-\mathrm{LiAlO}_{2}$ system. Oxynitride solid-electrolyte films were synthesized under an $\mathrm{N}_{2}$ atmosphere by reactive $\mathrm{RF}$ sputtering with cathodes made of different materials, such as $\mathrm{Li}_{3} \mathrm{PO}_{4}, \mathrm{Li}_{4} \mathrm{SiO}_{4}$, and $\mathrm{LiAlO}_{2}$ installed at different positions in the chamber. The formation of amorphous films with no grains and a continuous atomic distribution was confirmed using scanning electron microscopy and energy-dispersive X-ray spectroscopy. In a single synthesis, we fabricated an oxynitride solid-electrolyte film, in which the composition range of the components was approximately one-eighth that in the corresponding pseudoternary system. The highest Li-ion conductivity of $3.1 \times 10^{-6} \mathrm{~S} \mathrm{~cm}^{-1}$ was obtained for the LiPSiAlON film with a composition ratio of LiPON : LiSiON : LiAION $=48.5$ : 33.9 : 17.6. The different bonding states of $\mathrm{O}$ and $\mathrm{N}$ in the LiPSiAlON film doped with $\mathrm{P}, \mathrm{Si}$, and $\mathrm{Al}$ were examined using X-ray photoemission spectroscopy. The ionic conductivity was improved by the mixed anion effect. The combinatorial synthesis enables the efficient optimization of the chemical composition and facilitates the development of highly conductive amorphous solid electrolytes.

(c) The Author(s) 2022. Published by ECSJ. This is an open access article distributed under the terms of the Creative Commons Attribution-NonCommercialShareAlike 4.0 License (CC BY-NC-SA, http://creativecommons.org/licenses/by-nc-sa/4.0/), which permits non-commercial reuse, distribution, and reproduction in any medium by share-alike, provided the original work is properly cited. For permission for commercial reuse, please email to the corresponding author. [DOI: 10.5796/electrochemistry.22-00002].

Keywords : Thin Film, Solid Electrolyte, Combinatorial Synthesis, Lithium Batteries

\section{Introduction}

All-solid-state thin-film secondary Li batteries (TFBs) are viable for use as auxiliary power sources in small devices, such as wireless 3 sensors and smart devices. TFBs, which are mainly fabricated by 4 physical vapor deposition, consist of a cathode, solid electrolyte, 5 metal anode, and current collectors arranged in a layer. ${ }^{1,2}$ Generally, 6 a solid electrolyte layer is deposited on the cathode layer at room 7 temperature to suppress undesirable chemical reactions that generate 8 an ionically resistive interphase. ${ }^{3}$ Amorphous solid-electrolyte 9 layers have a uniform morphology with no grain boundaries, which suppresses the short circuit between the cathode and anode layers. 1 Due to this advantage, amorphous materials have been used as solid electrolytes in this study. Amorphous Li phosphorus oxynitride 3 (LiPON) has been widely used for the fabrication of solid electrolyte 4 layers owing to its relatively high $\mathrm{Li}$ ionic conductivity $\left(\sim 10^{-6}\right.$ ${ }_{5} \mathrm{~S} / \mathrm{cm}$ at $25^{\circ} \mathrm{C}$ ) and superior electrochemical stability, ${ }^{4}$ compared to 6 other amorphous materials, such as $\mathrm{Li}_{3} \mathrm{PO}_{4}, \mathrm{Li}_{2} \mathrm{SiO}_{3}, \mathrm{Li}_{4} \mathrm{SiO}_{4}$, and 7 Li-Si-V-O glass. ${ }^{5-7}$ However, the ionic conductivity of LiPON is 8 much lower than those of crystalline solid electrolytes composed of ${ }_{9}^{9}$ perovskite- and garnet-type oxides, ${ }^{8}$ which limits the high-power

${ }^{1}{ }^{1}$ ECSJ Active Member

$2 \S^{\S}$ ECSJ Fellow

S. Sasaki (D) orcid.org/0000-0001-9683-631X

${ }^{4}$ K. Suzuki (D) orcid.org/0000-0002-2473-0724

5 M. Hirayama (D) orcid.org/0000-0003-4804-4208

6 R. Kanno (D) orcid.org/0000-0002-0593-2515 operation of TFBs. To improve the apparent ionic conductivity, it is necessary to decrease the thickness of the solid electrolyte layer and increase the electrochemical reaction area. To address this technical challenge associated with TFB fabrication, it is important to develop amorphous electrolytes with higher $\mathrm{Li}$ ionic conductivities. Tatsumisago et al. reported that the $\mathrm{Li}$ ionic conductivities of amorphous $\mathrm{Li}$ phosphate can be improved by adding $\mathrm{Li}$ silicate because of the mixed anion effect. ${ }^{9}$ Although the mixed anion effect has been proposed by other research groups, ${ }^{10-18}$ the synthetic conditions and equipment are different in each report. Therefore, it is difficult to discuss the detailed mechanism of the mixed anion effect and the corresponding optimal chemical composition in a unified manner.

Combinatorial synthesis is an efficient method for accessing material libraries. It is an experimental method for efficiently synthesizing multiple varieties of materials using the same synthetic route. The technology of combinatorial synthesis was proposed in the 1960 s by Merrifield ${ }^{19}$ (solid-phase peptide synthesis). The application of this technology for the development of suitable materials begun in the $1990 \mathrm{~s} .{ }^{20}$ In particular, it has led to the discovery of new active substances, ${ }^{21}$ which has had an impact on the pharmaceutical industry. Beal et al. ${ }^{22}$ developed a perovskitetype solid electrolyte $\left(\mathrm{Li}_{3 x} \mathrm{La}_{2 / 3-x} \mathrm{TiO}_{3}, \quad x=0.11\right)$ through a combinatorial evaporation synthesis method using an E-beam or a 62 Knudsen cell.

Radio Frequency (RF) sputtering is one of the most suitable 6 methods for synthesizing electronically insulating oxide thin films. ${ }^{23}$ When an ionized gas with high energies of $>10 \mathrm{eV}$ collides with the 66 
1 target material, the species of the target material simultaneously 2 scatter from the surface and deposits onto the opposing substrate. 3 This allows for a highly reproducible and fast deposition of 4 materials with high melting points, and the chemical composition of 5 the deposited films is relatively close to that of the target material, 6 compared to vapor deposition and electron beam evaporation. 7 However, there have been no reports on the combinatorial synthesis 8 of solid electrolyte materials using RF sputtering. Vacuum film 9 deposition via combinatorial synthesis methods using RF sputtering 0 can be achieved through judicious equipment design and the 1 incorporation of unique sputtering technologies. In this study, we 2 developed a combinatorial synthesis system for the simultaneous 3 deposition of multiple materials using RF sputtering with different 4 targets. Oxynitride solid-electrolyte films were fabricated in pseudo5 binary LiPON-LiSiON, LiSiON-LiAlON, and LiPON-LiAlON 6 systems, and pseudo-ternary LiPON-LiSiON-LiAlON systems, 7 and their structures and $\mathrm{Li}$ ionic conductivities were investigated.

\section{Materials and Methods}

Oxynitride solid electrolyte films were fabricated using a combinatorial reactive RF system (QAM-4ST, ULVAC), as shown 3 in Fig. 1. The system is equipped with four sputtering cathodes and 24 a substrate holder in the process chamber for the simultaneous 5 deposition of materials. All the sputtering cathodes were positioned 26 outwards relative to the center of the substrate. Each sputtering 27 target set to the cathode was tilted at an angle of $30^{\circ}$ to the center. 28 The distance between the center of the target and the substrate 2 changed from 150 to $215 \mathrm{~mm}$, depending on the substrate position. When the substrate was rotated during deposition, the deposited film 31 had a uniform thickness distribution of less than $\pm 5 \%$ within the 2 substrate with a diameter of $101.6 \mathrm{~mm}$.

In contrast, an atomic distribution was observed in the film 34 deposited with the fixed substrate. To operate the multiple RF 5 cathodes simultaneously, it is essential to suppress the interference 36 of the RF circuits, including the matching box between the RF 37 cathodes, because the interference causes the application of a non38 uniform power balance to each RF cathode and finally leads to 9 a mismatch between the incident and reflected waves. The RF 40 frequency is typically shifted by several $\mathrm{kHz}$ to suppress 1 interference. However, the effective output remains unclear because 2 the reflected wave cannot be completely suppressed. In our system, ${ }_{3} 3$ all the power supplies are connected to the phase shifter in the RF 4 cathodes, enabling control of the phase of the output transmitted 5 from the RF power supply. It is configured such that the power 46 balance applied by the phase difference of each RF power supply 7 can be controlled. Additionally, such a configuration ensures 8 alignment of the power balance from the matching box to the 9 sputtering target, special design of the distance, and phase adjust5 ment. As a result, the simultaneous deposition of stable films is 1 possible with multiple RF cathodes.

The process chamber was evacuated to a pressure of below $31 \times 10^{-4} \mathrm{~Pa}$ using a turbo molecular pump and a rotary pump. The 54 oxynitride solid electrolyte films were fabricated using different 5 cathodes under an $\mathrm{N}_{2}$ atmosphere. $\mathrm{Li}_{3} \mathrm{PO}_{4}, \mathrm{Li}_{4} \mathrm{SiO}_{4}, \mathrm{LiAlO}_{2}$ pellets 6 were used as the targets and the films were deposited on glass 7 substrates (diameter $101.6 \mathrm{~mm}$, Eagle XG Corning). Pt and Ti films 8 of 100 and $200 \mathrm{~nm}$ thicknesses, respectively, were deposited as 9 lower electrodes on the substrate surface. The solid electrolyte films o were deposited on the metal electrode film at an $\mathrm{N}_{2}$ pressure of $10.3 \mathrm{~Pa}$ and power output of 100 or $200 \mathrm{~W}$. Two or three reagent 2 materials were simultaneously deposited on the substrate using 3 different RF cathodes. The positions of the targets and substrates 64 were fixed during deposition. This introduced a gradual slope in the 5 chemical composition and thickness of each solid-electrolyte film. 6 After the deposition of the solid-electrolyte films, Pt upper electrode
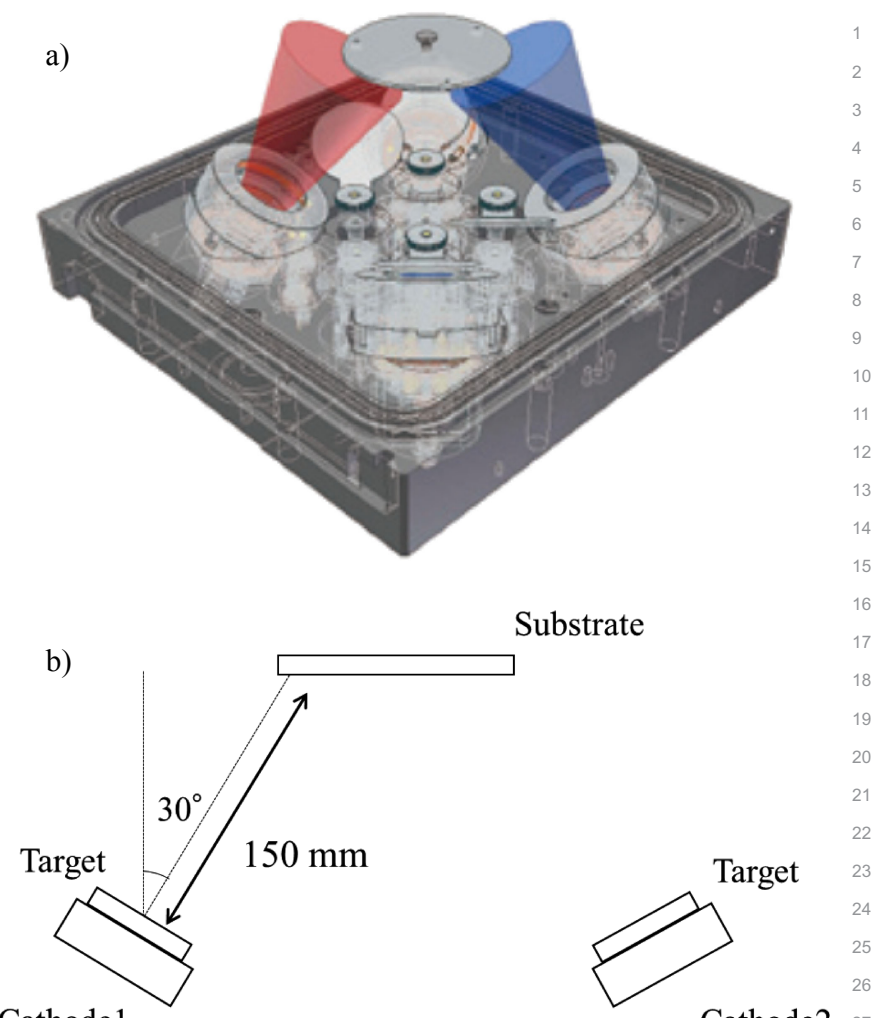

Substrate

Cathode1

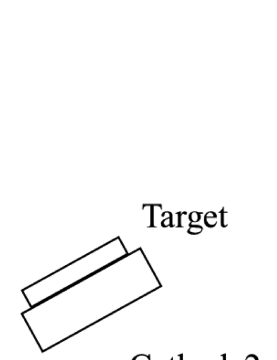

c)

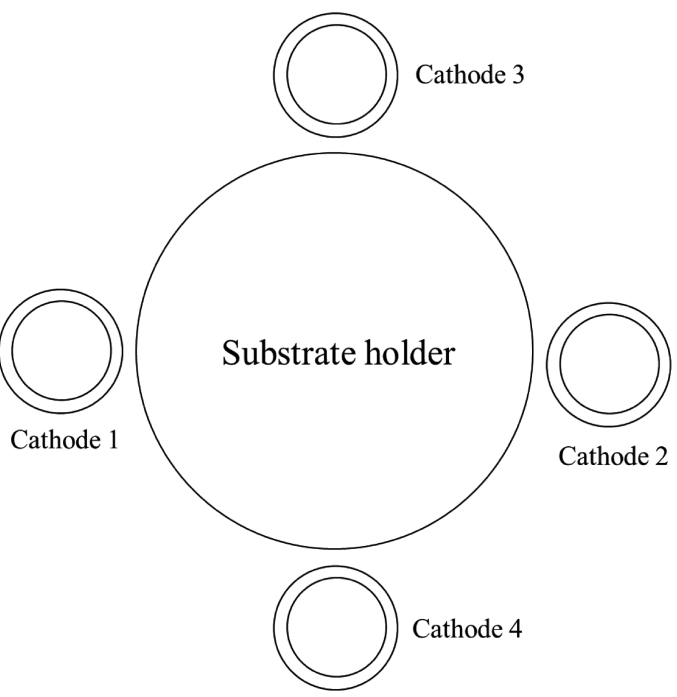

Cathode2

Figure 1. Schematics of RF sputtering system for combinatorial synthesis. a) overall view, b) cross-sectional view, and c) top view.

films with diameters of $5 \mathrm{~mm}$ were deposited in an X-pattern onto 52 the films.

The film thickness of the solid electrolyte films was measured 54 using a profilometer (DEKTAK-XT Bruker) and an ellipsometer 55 (ESM-1AT ULVAC). The deposition rate of each film was 56 calculated based on the film thickness and duration of deposition. 57 The film surface conditions and composition distributions were 58 measured using scanning electron microscopy (SEM, SU8220, 59 Hitachi High-tech) and energy dispersive X-ray (EDX, 60 Xflash5060FQ, Bruker) analysis. Structural investigations of 61 the films were performed using Raman spectroscopy (inVia, 62 RENISHAW) and X-ray diffraction (XRD, Smartlab, Rigaku) and 63 X-ray photoelectron spectroscopy (XPS, VersaProbe III, ULVAC- 64 PHI). XPS spectra were recorded for the Li 1s, P 2p, Si 2p, O 1s, 65 and $\mathrm{N} 1 \mathrm{~s}$ transitions. The X-ray source was $\mathrm{Al} \mathrm{K \alpha}(1486.6 \mathrm{eV})$, and 66 
1 the beam diameter was $100 \mu \mathrm{m}(100 \mathrm{~W}, 20 \mathrm{kV})$. The signal 2 extraction angle, path energy, and step size were $45^{\circ}, 55 \mathrm{eV}$, and $0.10 \mathrm{eV}$, respectively.

The electrochemical properties of the films were investigated via 5 AC impedance spectroscopy between the upper and lower $\mathrm{Pt}$ 6 electrodes using an electrochemical measuring instrument (IM6 7 Zahner). An AC voltage of $10 \mathrm{mV}$ was applied in the frequency 8 range of $0.1 \mathrm{~Hz}$ to $1 \mathrm{MHz}$ at room temperature. The ionic 9 conductivities were calculated using the electrode area $\left(25 \mathrm{~mm}^{2}\right)$, o film thickness, and resistance determined using impedance spec1 troscopy. The film thicknesses of LiPSiON, LiSiAlON, LiPAlON, 2 and LiPSiAlON were estimated from the deposition rates of the LiPON, LiSiON, and LiAlON single films determined from the 4 thicknesses at each position on the substrate.

\section{Results and Discussion}

\subsection{Combinatorial synthesis of Li conductive films in pseudo-} binary systems

LiPON and LiAlON single films were deposited on a fixed Pt/Ti 1 substrate for $2 \mathrm{~h}$ to investigate the position dependence of the film 2 thickness. The $\mathrm{Li}_{3} \mathrm{PO}_{4}$ and $\mathrm{LiAlO}_{2}$ targets were set on cathodes 1 3 and 2, as shown in Fig. 1, and were deposited under an $\mathrm{N}_{2}$ 24 atmosphere at 200 and $100 \mathrm{~W}$, respectively. The film thicknesses 5 were measured at 15 positions with an interval of $6.25 \mathrm{~mm}$ for each 26 sample. Figure 2 shows the position dependence of the thicknesses 27 of the LiPON and LiAlON single films deposited on the Pt/Ti 28 substrate. The deposited films had the largest thickness at the 29 position nearest to the sputtering cathode. The thickness of the LiPON film at position 1 was $341 \mathrm{~nm}$, which corresponded to a 3 deposition rate of $2.84 \mathrm{~nm} \mathrm{~min}^{-1}$. The film thickness gradually 32 decreased to $46 \mathrm{~nm}$ at position 15 as the distance from the target 3 increased. The thickness of the LiAlON film at position 1 was $3434 \mathrm{~nm}$, which gradually increased to $100 \mathrm{~nm}$ at position 15 as the 5 distance from the target decreased. The deposition rate of the LiAlON film was $0.830 \mathrm{~nm} \mathrm{~min}^{-1}$ at position 15 , which was much 37 lower than that of the LiPON film. The proportion of each material 38 can be changed by changing the power of each cathode. From the 9 thicknesses of the LiPON and LiAlON films observed at each position, we estimated the composition ratio of LiPON to LiAlON in 1 the LiPON-LiAlON film fabricated via simultaneous deposition

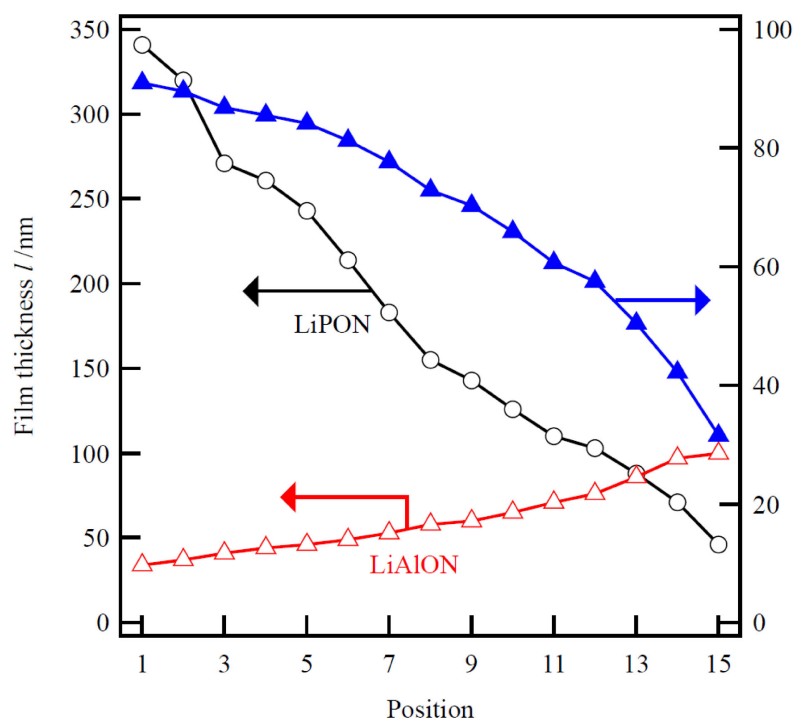

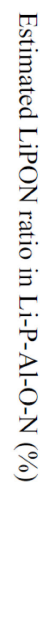

3 Figure 2. Position dependence of thicknesses of LiPON and 64 LiAlON single films deposited on Pt/Ti substrate for $120 \mathrm{~min}$ and 5 estimated composition ratio of LiPON to LiAlON in LiPAlON film 6 fabricated by simultaneous deposition. using multiple cathodes. The estimated composition ratio in the LiPON-LiAlON film is shown in Fig. 2. The ratios of LiPON to LiAlON were 91.0 and $31.6 \%$ at positions 1 and 15, respectively. A gradual decrease in the ratio was observed from position 1 to 15 , which indicated the formation of the LiPON-LiAlON film with a continuous atomic distribution. This result demonstrates the feasibility of combinatorial synthesis of the LiPON-LiAlON film with a continuous compositional distribution.

With the same positions of the substrate and targets, the LiPAlON film was synthesized by the simultaneous deposition of the LiPON and LiAlON targets. Figure 3 shows the Raman spectra of the LiPAlON films with different ratios of LiPON to LiAlON. Three major peaks were observed at approximately 800, 960, and $1020 \mathrm{~cm}^{-1}$, which were attributed to $\mathrm{P}-\mathrm{N}=\mathrm{P}, \quad \mathrm{PO}_{4}{ }^{3-}$, and 14 $\mathrm{P}_{2} \mathrm{O}_{7-x} \mathrm{~N}_{x}{ }^{n-}$, respectively. The $\mathrm{PO}_{4}{ }^{3-}$ peak was observed at all the measurement points, which is consistent with the existence of 16 LiPON at all the positions, as shown in Fig. 2.

Although the LiPON content decreased gradually from position 1 to 15 , position 6 exhibited the lowest intensity of the $\mathrm{PO}_{4}{ }^{3-}$ peak. This result indicates that the introduction of LiAlON alters the chemical bonding between $\mathrm{P}$ and $\mathrm{O}$. Figure 4 depicts the SEM and EDX images of the LiPON-LiAlON film collected in the region 22 between positions 8 and 9. The SEM image shows no significant 23 changes in the contrast, which indicates a very smooth surface with 24 no grain boundaries. The EDX analyses detected $\mathrm{O}, \mathrm{P}$, and $\mathrm{Al} 25$ signals, and all the elements were uniformly distributed in the film 26 without segregation. This result indicates the formation of an 27 amorphous LiPAlON film with a continuous atomic distribution.

Figure 5 shows the room-temperature $\mathrm{Li}$ ionic conductivities 29 of the LiPAlON films with different compositions. The ionic 30 conductivities were calculated from the estimated thicknesses and 3 resistance values of the first semicircles in the Nyquist plots, shown 32 in the insets in Fig. 5. The film compositions at each position were 33 estimated from the deposition rates of the LiPON and LiAlON 34 single films on the Pt/Ti substrate (Fig. 2).

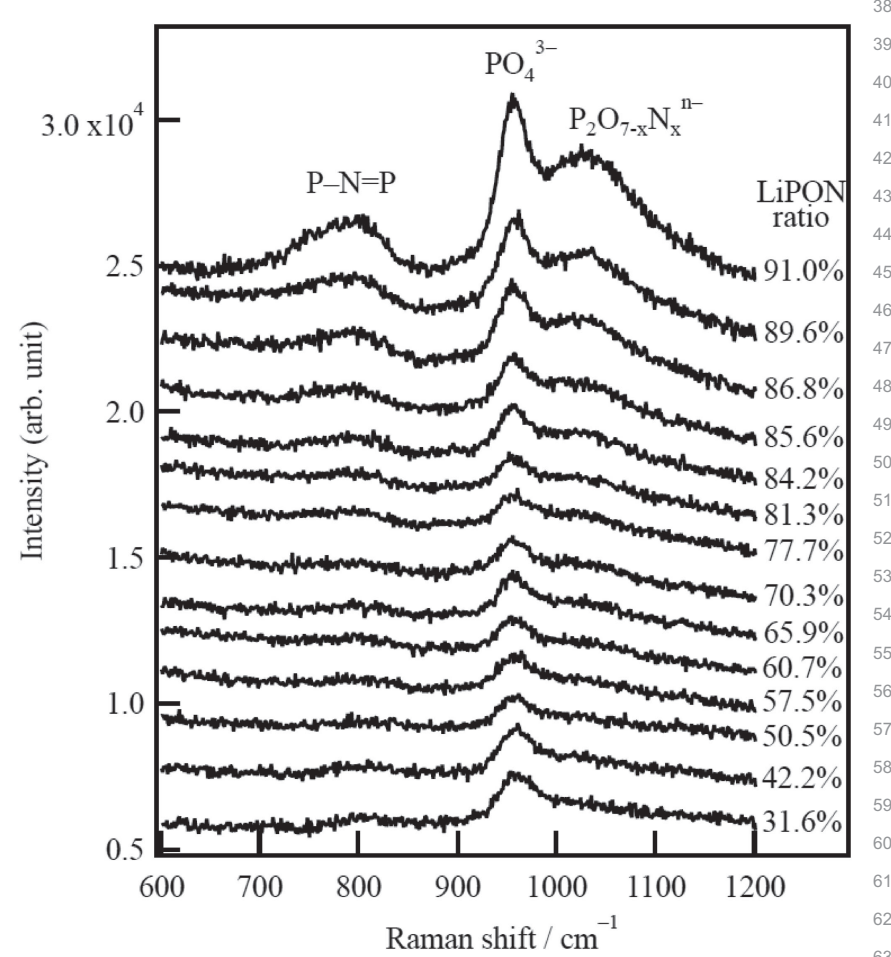

Figure 3. Raman spectra of LiPAlON films with different ratios 64 of LiPON to LiAlON fabricated at different positions on $\mathrm{Pt} / \mathrm{Ti} 65$ substrate. The measurement positions are shown in Fig. 2. 

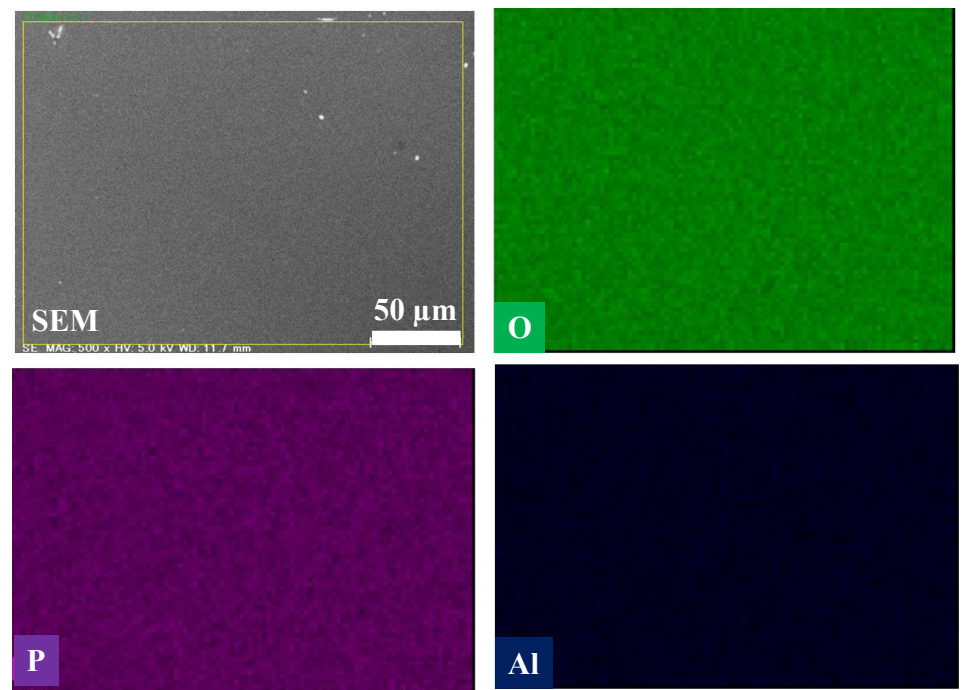

Figure 4. SEM-EDX images of the LiPON-LiAlON film between positions 8 and 9 .

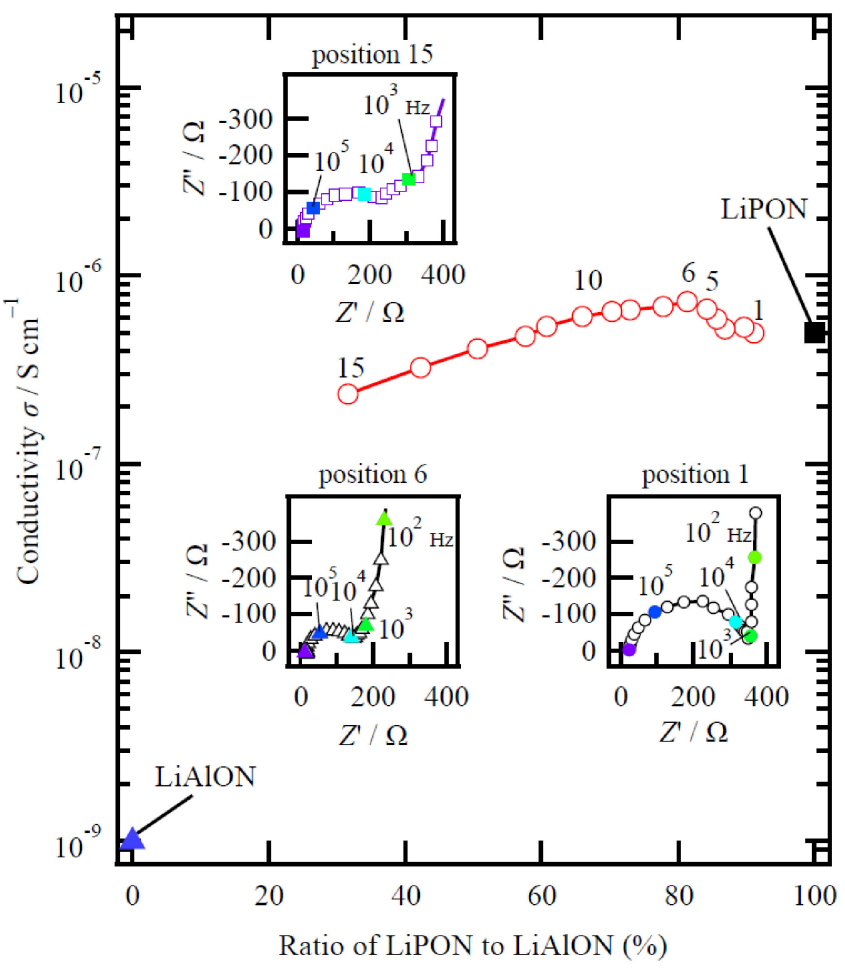

Figure 5. Ionic conductivity changes of the LiPON-LiAlON films depending on the film composition. The film compositions were estimated by their deposition rates at each position on the substrate. Insets depict the Nyquist plots of the films with the LiPON/LiAlON ratios of $91.0,81.3$, and $31.6 \%$. Ionic conductivities of end-member single films are presented for comparison.

The ionic conductivities of the LiPON and LiAlON single films $\left(5.0 \times 10^{-7} \mathrm{~S} \mathrm{~cm}^{-1}\right.$ and $1.0 \times 10^{-9} \mathrm{~S} \mathrm{~cm}^{-1}$, respectively) were also 9 plotted for comparison. The conductivity showed no significant change when the ratio of LiPON to LiAlON was in the range of 100 1 to $86.8 \%$. When the ratio decreased from 86.8 to $81.3 \%$, the ionic 2 conductivity increased slightly to $7.3 \times 10^{-7} \mathrm{~S} \mathrm{~cm}^{-1}$. In the Raman 3 spectra shown in Fig. 3, the $\mathrm{PO}_{4}{ }^{3-}$ peak had the lowest intensity 64 with the largest peak width at the position with a LiPON content of $581.3 \%$. Upon reducing the LiPON content to $31.6 \%$, the ionic 6 conductivity decreased to $2.4 \times 10^{-7} \mathrm{~S} \mathrm{~cm}^{-1}$ owing to the higher proportion of the poorly conductive LiAlON phase. Randomizing 23 the binding state of $\mathrm{PO}_{4}{ }^{3-}$ facilitated the $\mathrm{Li}$ ionic conduction in the 24 amorphous LiPAION film. The change in the local structures around 25 the $\mathrm{Li}$ ions was inferred to be a consequence of the mixed anion 26 effect. According to a previous report, ${ }^{24}$ amorphous solid electro- 27 lytes contain stable sites where the potential energy of the Li ions is 28 low because of the surrounding atoms and their bonding states, and 29 the $\mathrm{Li}$ ions are conducted via the pathways with the lowest 30 activation energies from one stable site to another. The configura- 3 tional freedom of the diffusion pathways in amorphous solid 32 electrolytes formed by multiple ortho-oxoanions, such as $\mathrm{MO}_{x}{ }^{-}$ $(\mathrm{M}=\mathrm{P}, \mathrm{Si}$, and $\mathrm{B})$, is significantly higher than that of those 34 comprising a single type of ortho-oxoanions. Therefore, it is 35 probable that diffusion occurs at lower energies in the presence of 36 multiple ortho-oxoanions. Although certain experimental results 37 support the feasibility of using different types of anions for 38 improving the ionic conductivity, a guiding principle for mixing 39 different types of anions for this purpose has not been established.

The LiPSiON and LiSiAlON films were fabricated using the 41 same method as that used for the LiPAlON film (all the films were 42 deposited at a power of $100 \mathrm{~W}$ ), and their ionic conductivities were 43 investigated. Figure 6 shows the ionic conductivities of the 44 LiPSiON and LiSiAlON films with different compositions. The 45 chemical compositions were estimated based on the deposition rates 46 of each target at different positions on the substrate. In the LiPON- 47 LiSiON system, the ionic conductivity exceeded $10^{-6} \mathrm{~S} \mathrm{~cm}^{-1}$ when 48 the LiPON to LiSiON ratio ranged from 20.9 to $92.9 \%$. Lower ionic 49 conductivities were observed for the end components, LiPON 50 $\left(5.0 \times 10^{-7} \mathrm{~S} \mathrm{~cm}^{-1}\right)$ and LiSiON $\left(6.2 \times 10^{-7} \mathrm{~S} \mathrm{~cm}^{-1}\right)$. The results at 51 positions 1 and 15 showed that mixing small amounts of content 52 increased ionic conductivity. This observation is consistent with a 53 previous report. ${ }^{17}$ In the LiSiON-LiAlON system, the composition 54 ratio of $\mathrm{LiSiON}$ to LiAlON changed from 11.2 to $73.0 \%$. In contrast 55 to the LiPAlON and LiPSiON films, the ionic conductivities were 56 lower than that of the LiSiON single film. According to our 57 experimental results, the ionic conductivities of the LiPON-based 58 solid electrolytes can be changed by randomizing the binding state 59 of each element through the mixing of multiple anion species.

\subsection{Combinatorial synthesis in a pseudo-ternary $\mathrm{Li}_{3} \mathrm{PO}_{4}$ $\mathrm{Li}_{4} \mathrm{SiO}_{4}-\mathrm{LiAlO}_{2}$ system}

To examine the effect of mixing three anionic species on the $\mathrm{Li} 64$ conductivity, we synthesized LiPSiAlON films based on a pseudo- 65 ternary system using two different targets, LiSiON and LiPON- 66 
(a)

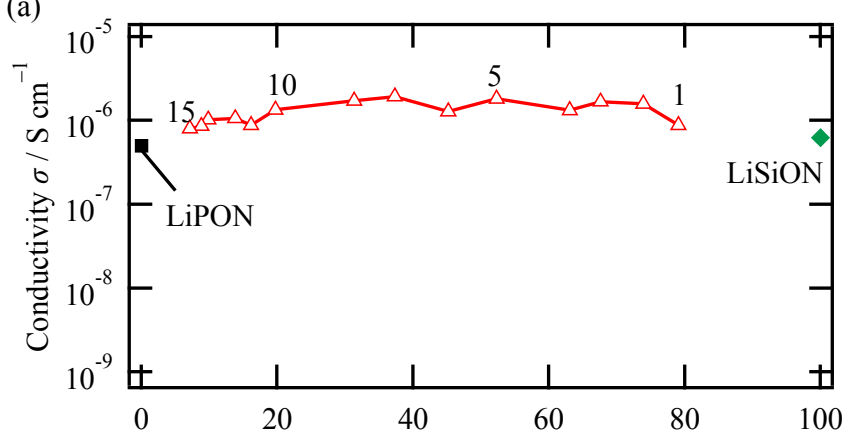

(b)

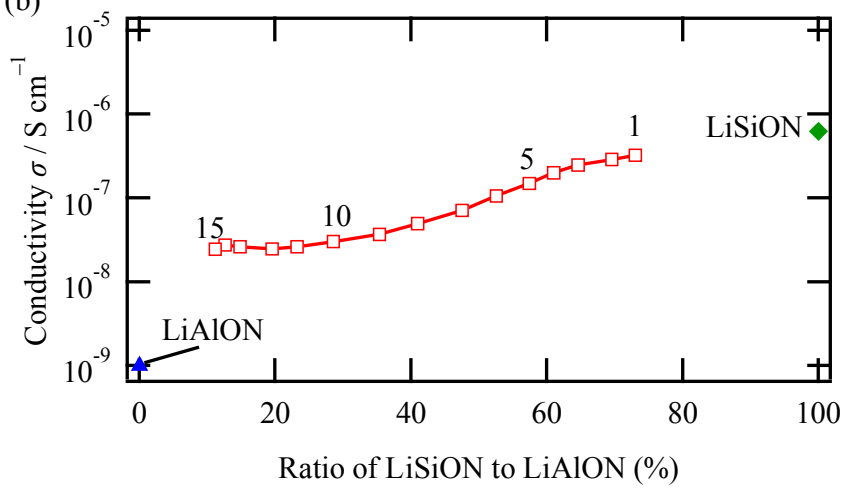

Figure 6. Li ionic conductivities of (a) LiPON-LiSiON and (b) LiSiON-LiAlON films with different chemical compositions. Ionic conductivities of end-member single films are also depicted for comparison.

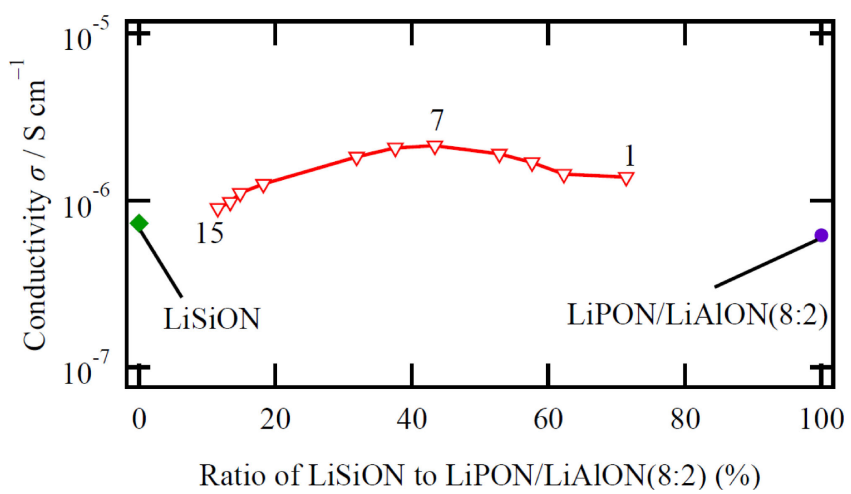

Figure 7. Variation of $\mathrm{Li}$ ionic conductivity with chemical compositions of LiPAlON-LiSiON film. Ionic conductivities of end-member single films are also depicted for comparison.

LiAlON (molar ratio: $8: 2$ ). The films were synthesized by simultaneous deposition using $\mathrm{Li}_{4} \mathrm{SiO}_{4}$ and $\mathrm{Li}_{3} \mathrm{PO}_{4}: \mathrm{LiAlO}_{2}(8: 2)$ 5 targets which were positioned such that they faced each other. 36 Figure 7 shows the variation in the ionic conductivity with the 7 chemical compositions of the LiPSiAlON films. The film showed a 8 higher ionic conductivity than the end components, LiSiON and 9 LiPAlON ( $\mathrm{P}: \mathrm{Al}=8: 2)$, for all the compositions. The highest conductivity of $2.1 \times 10^{-6} \mathrm{~S} \mathrm{~cm}^{-1}$ was observed when the compo51 sition ratio of $\mathrm{Li}_{4} \mathrm{SiO}_{4}$ to $\mathrm{Li}_{3} \mathrm{PO}_{4}: \mathrm{LiAlO}_{2}(8: 2)$ was $43.4 \%$, which 2 calculated to an LiPON : LiSiON : LiAlON ratio of $44.6: 42.7$ : 3 12.7. Although the LiPSiAlON films exhibited slightly improved 64 conductivity compared to those of the end-members, the conductiv5 ities were comparable to those of the LiPSiON film $\left(1.9 \times 10^{-6}\right.$ ${ }_{6} \mathrm{~S} \mathrm{~cm}^{-1}$ ) with no $\mathrm{Al}$ species. The LiSiON-LiPAlON $(\mathrm{P}: \mathrm{Al}=8: 2)$ a)

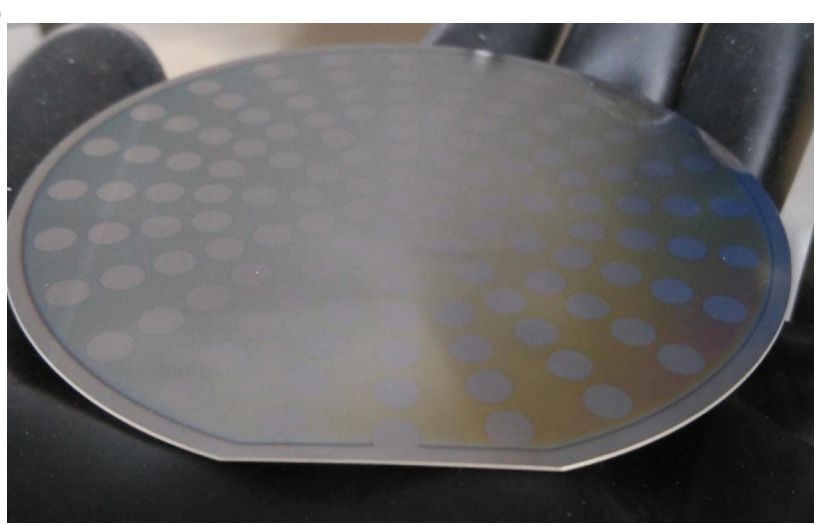

b) Cathode 1

Cathode 2

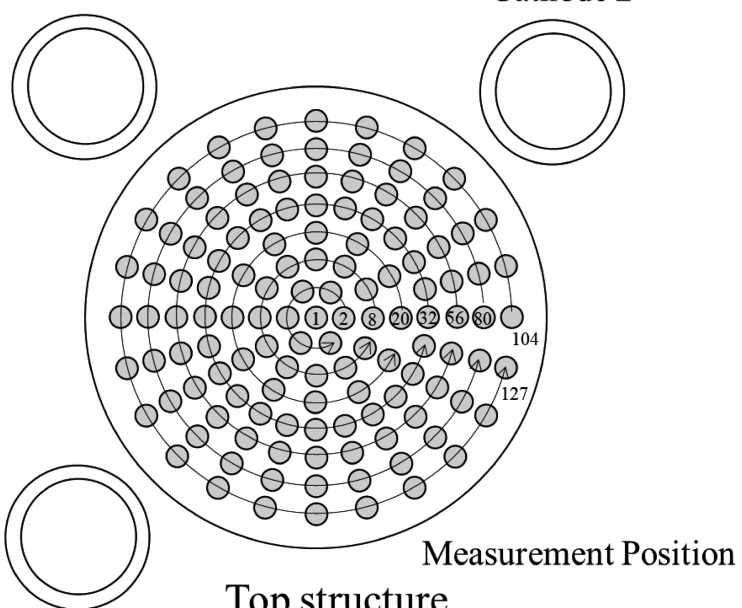

Cathode 3

Figure 8. Electrode positions and photograph of a LiPONLiSiON-LiAlON film with different composition ratios.

system showed no significant effect of Al doping. The LiPON : 4 LiSiON : LiAlON ratio of the LiPSiAlON film was analyzed to 41 $49.2: 39.3: 11.5$ from XPS peak fitting. This value is consistent 42 with the calculated composition of $44.6: 42.7: 12.7$ using the 43 deposition rates of the single films synthesized with each target. 44 Thus, the film compositions of the combinatorially synthesized films 45 were estimated from the deposition rates in this study.

To efficiently investigate LiPSiAlON films with a wider 47 compositional range, we fabricated an LiPSiAlON film by 48 simultaneous sputtering under an $\mathrm{N}_{2}$ atmosphere using three cathode 49 targets: $\mathrm{Li}_{3} \mathrm{PO}_{4}, \mathrm{Li}_{4} \mathrm{SiO}_{4}$, and $\mathrm{LiAlO}_{2}$. Figure 8 a shows the setting 50 positions of the substrate and three cathode targets in the chamber 51 and a photograph of the LiPSiAlON film. A total of 127 positions 52 were selected to investigate the compositions and ionic conductiv- 53 ities of the LiPSiAlON film. According to Fig. 8b, different color 54 distributions were observed depending on the position due to the 55 interference of light induced by the differences in film thickness and 56 refractive index. The composition ratios in the LiPSiAlON films 57 were estimated from the deposition rates of the LiPON, LiSiON, and 58 LiAlON single films. The film thicknesses and composition ratios 59 corresponding to all the positions are listed in Table S1 (Supple- 60 mentary Information). The contents of LiPON, LiSiON, and 61 LiAlON were 28.8 to $57.5 \%, 14.7$ to $49.2 \%$, and 17.0 to $48.2 \%$, 62 respectively. Figure 9 shows the XRD patterns of the LiPON : 63 LiSiON : LiAlON films with different LiPON : LiSiON : LiAlON 64 ratios of $31.4: 49.2: 19.4,57.2: 17.5: 25.3$, and $43.4: 15.0: 41.6$. 65 All XRD patterns showed the 111 diffraction peaks of the $\mathrm{Pt} 66$ 


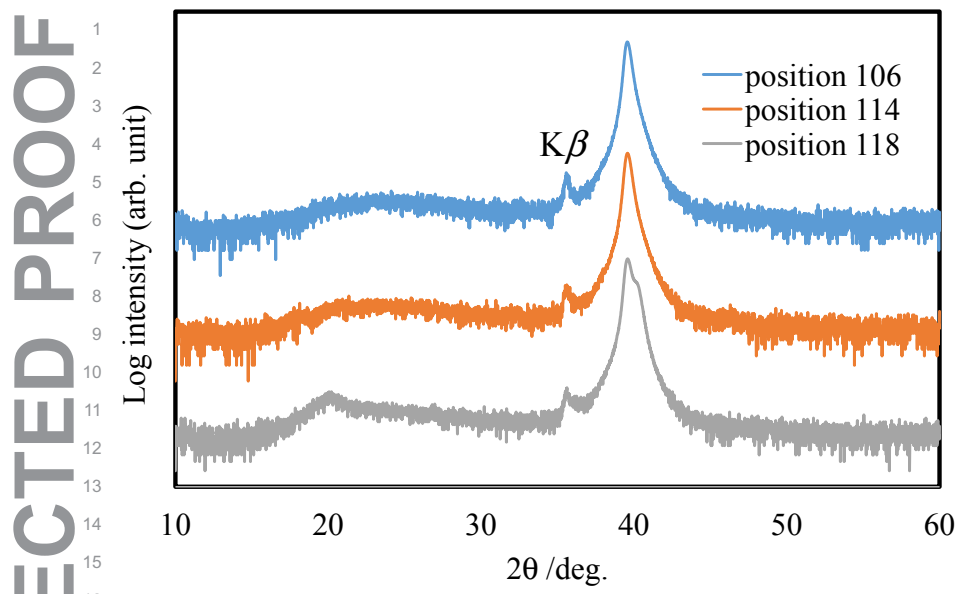

Figure 9. XRD patterns of LiPSiAlON films in ternary LiPONLiSiON-LiAlON system at position 106, 114, and 118.

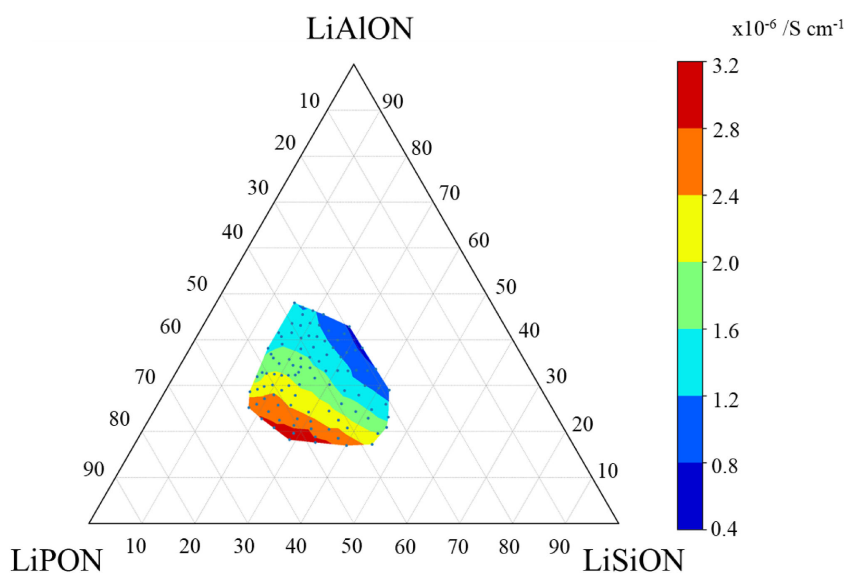

Figure 10. Ionic conductivities of LiPSiAlON films in ternary LiPON-LiSiON-LiAlON system.

substrate at around $40^{\circ}$ and very broad peaks between $15^{\circ}$ and $30^{\circ}$. The broad peaks confirmed that the amorphous LiPON : LiSiON : 4 LiAlON films were formed regardless of the film composition.

Figure 10 illustrates the dependence of the ionic conductivity of 46 the LiPSiAlON film on the film position. The ionic conductivity 7 gradually changes with the position depending on the film 8 composition. Compared with the films obtained by combinatorial 9 synthesis using two materials, the measurable range changed from linear to areal, and the maximum value $\left(3.1 \times 10^{-6} \mathrm{~S} \mathrm{~cm}^{-1}\right)$ of the 1 ionic conductivity corresponded to position 111 where the LiPON : 2 LiSiON : LiAlON ratio was 53.1 : 28.7 : 18.2.

Figure 11 shows the X-ray photoelectron spectra of the ${ }_{4}$ LiPSiAlON film collected at position 111, where the film showed 55 the highest ionic conductivity. The binding energies of the Li $1 \mathrm{~s}$ and 6 P 2 p orbitals were 55.3 and $133.1 \mathrm{eV}$, respectively, which were not 7 significantly different from the corresponding values for the LiPON 5 film. In contrast, the $\mathrm{Si} 2 \mathrm{p}$ peak shifted from $102.5 \mathrm{eV}$ for the $9 \mathrm{LiSiON}$ film to $101.7 \mathrm{eV}$ for the LiPSiAlON film. The binding so energy of Si was decreased upon doping with $\mathrm{P}$ and $\mathrm{Al}$. The $\mathrm{O}$ 1s 51 peak of the LiPSiAlON film was observed at $531.4 \mathrm{eV}$, which was 2 slightly different from those observed for LiPON $(531.2 \mathrm{eV})$ and 3 LiSiON $(531.8 \mathrm{eV})$. Furthermore, two $\mathrm{N}$ 1s peaks were observed 64 at 398.2 and $397.2 \mathrm{eV}$, corresponding to LiSiON and LiPON, 55 respectively. These results suggest that the $\mathrm{O}$ and $\mathrm{N}$ atoms in the 6 LiPSiAlON film had intermediate binding conditions to those in the
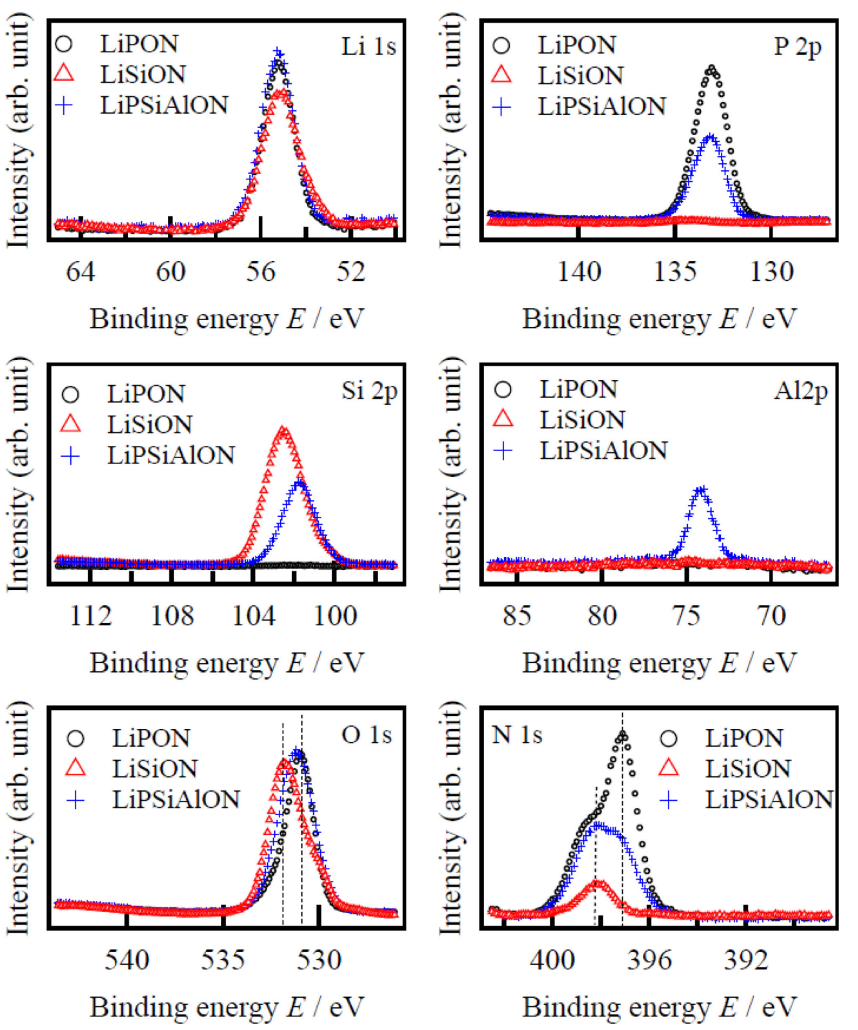

Figure 11. $\mathrm{Li} 1 \mathrm{~s}, \mathrm{P} 2 \mathrm{p}, \mathrm{Si} 2 \mathrm{p}, \mathrm{Al} 2 \mathrm{p}, \mathrm{O} 1 \mathrm{~s}$, and $\mathrm{N} 1 \mathrm{~s}$ X-ray photoemission spectra of the LiPSiAlON film. The spectra obtained for the LiPON and LiSiON films are also shown for comparison.

LiPON and LiSiON films. It is postulated that the intermediate 34 conditions affect the diffusion pathways of the Li ions. From the 35 quantitative analyses of the XPS peaks, the chemical formulas 36 for the LiPON, LiSiON, and LiPSiAlON species were estimated 37 to be $\mathrm{Li}_{1.90} \mathrm{PO}_{3.01} \mathrm{~N}_{0.61}, \quad \mathrm{Li}_{2.12} \mathrm{SiO}_{4.34} \mathrm{~N}_{0.13}$, and $\mathrm{Li}_{1.88} \mathrm{P}_{0.51} \mathrm{Si}_{0.42^{-}}$ $\mathrm{Al}_{0.07} \mathrm{O}_{3.46} \mathrm{~N}_{0.36}$, respectively. All the materials showed a decrease in Li composition compared to the target. Notably, LiPSiAlON, with 40 the highest ionic conductivity, had the lowest Li content. The ionic 41 conductivity is generally proportional to the product of the carrier 42 concentration and mobility. Therefore, the high ionic conductivity 43 observed in films with low Li contents should be associated with the 44 improved mobility induced by the mixed anion effect. However, 45 there are no clear design guidelines for optimizing the mixed anion 46 effect to achieve high Li-ion conduction. The combinatorial 47 synthesis method is advantageous for efficiently synthesizing films 48 and identifying trends in the mixed anion effect in glassy solid 49 electrolytes. The ionic conductivity of the LiPSiAlON film was 50 further improved by increasing the carrier concentration of Li.

\section{Conclusion}

An efficient material search method was successfully developed 5 using combinatorial synthesis in conjunction with the co-sputtering 56 method to develop amorphous oxynitride solid electrolytes. In the ${ }_{57}$ pseudo-ternary $\mathrm{Li}_{3} \mathrm{PO}_{4}-\mathrm{Li}_{4} \mathrm{SiO}_{4}-\mathrm{LiAlO}_{2}$ system, a high ionic con- 5 ductivity of $3.1 \times 10^{-6} \mathrm{~S} \mathrm{~cm}^{-1}$ was obtained for the LiPSiAlON 59 film with a $\mathrm{LiPON}: \mathrm{LiSiON}: \mathrm{LiAlON}$ ratio of $48.5: 33.9: 17.6$. 60 The high ionic conductivity was associated with the randomization 61 of the binding state of each element by mixing multiple cation and 62 anion species. The proposed system is advantageous for the efficient 63 development of glassy solid electrolytes with high ionic conductiv- 64 ity which can potentially be applied in TFBs. 


\section{Acknowledgments}

This work was partially supported by the Grant-in-Aid for Scientific Research on Innovative Areas [19H05785].

\section{CRediT Authorship Contribution Statement}

Shunsuke Sasaki: Conceptualization (Lead), Data curation (Lead), Formal analysis (Lead), Investigation (Lead), Methodology (Lead), Resources (Lead), Software (Equal), Supervision (Equal), Validation (Lead), Visualization (Lead), Writing original draft (Lead)

${ }^{11}$ Atsuo Ono: Formal analysis (Equal), Software (Lead)

Akiyoshi Suzuki: Formal analysis (Equal), Methodology (Equal), Software (Equal)

Masaki Takei: Methodology (Equal), Resources (Equal), Supervision (Lead)

Kota Suzuki: Project administration (Equal), Supervision (Equal), Visualization (Equal), Writing - review \& editing (Lead)

Masaaki Hirayama: Conceptualization (Lead), Data curation (Lead), Project administration (Lead), Resources (Lead), Supervision (Equal), Visualization (Equal), Writing - original draft (Equal), Writing - review \& editing (Lead)

Ryoji Kanno: Conceptualization (Lead), Funding acquisition (Lead), Project administration (Lead), Resources (Lead), Supervision (Lead), Writing - review \& editing (Lead)

\section{Data Availability Statement}

The data that support the findings of this study are openly available under the terms of the designated Creative Commons License in J-STAGE Data listed in D1 of References.

\section{Conflict of Interest}

The authors declare no conflict of interest in the manuscript.

\section{Funding}

Japan Society for the Promotion of Science: $19 \mathrm{H} 05785$

\section{References}

D1. S. Sasaki, A. Ono, A. Suzuki, M. Takei, K. Suzuki, M. Hirayama, and R. Kanno, J-STAGE Data, https://doi.org/10.50892/data.electrochemistry.19083950, (2022).

1. J. B. Bates, N. J. Dudney, G. R. Gruzalski, R. A. Zuhr, A. Choudhury, C. F. Luck, and J. D. Robertson, J. Power Sources, 43, 103 (1993).

2. T. Jimbo, P. Kim, and K. Suu, Energy Procedia, 14, 1574 (2012).

3. J. B. Bates, N. J. Dudney, B. Neudecker, A. Ueda, and C. D. Evans, Solid State 7 Ionics, 135, 33 (2000).

4. X. Yu, J. B. Bates, G. E. Jellison, Jr., and F. X. Hart, J. Electrochem. Soc., 144, 524 (1997).

5. N. Kuwata, N. Iwagami, J. Kawamura, Y. Tanji, Y. Matsuda, and J. Kawamura, 1 J. Electrochem. Soc., 157, A521 (2010).

6. A. Nakagawa, N. Kuwata, Y. Matsuda, and J. Kawamura, J. Phys. Soc. Jpn., 79, 12 98 (2010).

7. H. Ohtsuka and J. Yamaki, J. Appl. Phys., 28, 2264 (1989).

8. N. Kamaya, K. Homma, Y. Yamakawa, M. Hirayama, R. Kanno, M. Yonemura, T 14 Kamiyama, Y. Kato, S. Hama, K. Kawamoto, and A. Mitsui, Nat. Mater., 10, 68215 (2011).

9. Y. Sakurai, A. Sakuda, A. Hayashi, and M. Tatsumisago, Solid State Ionics, 182, 59 (2011).

10. S. Lee, J. Kim, and D. Shin, Solid State Ionics, 178, 375 (2007).

11. M. Tatsumisago and T. Minami, Mater. Chem. Phys., 18, 1 (1987).

12. N. Kuwata, J. Kawamura, K. Toribami, T. Hattori, and N. Sata, Electrochem. 20 Commun., 6, 417 (2004).

13. Y. Ito, K. Miyauchi, and T. Oi, J. Non-Cryst. Solids, 57, 389 (1983).

14. Y. Yoon, C. Park, J. Kim, and D. Shin, Solid State Ionics, 225, 636 (2012).

15. F. Wu, Y. L. Zheng, L. Li, G. Tan, R. Chen, and S. Chen, J. Phys. Chem. C, 117, ${ }^{2}$ 19280 (2013).

16. T. Famprikis, J. Galipaud, O. Clernens, B. Pecquenard, and F. Le Cras, ACS Appl. 25 Energy Mater, 2, 4782 (2019)

17. Y. Su, J. Falgenhauer, T. Leichtweiß, M. Geiß, C. Lupó, A. Polity, S. Zhou, J. Obel, D. Schlettwein, J. Janek, and B. K. Meyer, Phys. Status Solidi B, 254, 1600088 (2017)

18. S. H. Jee, J. Y. Oh, H. S. Ahn, D.-J. Kim, H. C. Wikle, III, S. H. Kim, and Y. S. 29 Yoon, J. Mater. Sci., 45, 1709 (2010).

19. R. B. Merrifield, J. Am. Chem. Soc., 85, 2149 (1963).

20. X.-D. Xiang, X. Sun, G. Briceño, Y. Lou, K.-A. Wang, H. Chang, W. G. WallaceFreedman, S.-W. Chen, and P. G. Schultz, Science, 268, 1738 (1995).

21. M. J. Plunkett and J. A. Ellman, Sci. Am., 276(4), 68 (1997).

22. M. S. Beal, B. E. Hayden, T. Le Gall, C. E. Lee, X. Lu, M. Misaneh, C. Mormiche, D. Pasero, D. C. A. Smith, A. Weld, C. Yada, and S. Yokoishi, ACS Comb. Sci., 13, 375 (2011).

23. P. D. Davidse and L. I. Maissel, J. Appl. Phys., 37, 574 (1966).

24. T. Minami, J. Non-Cryst. Solids, 73, 273 (1985). 